\title{
Pourquoi j'ai accepté de témoigner dans le procès pour diffamation intenté par Hubert Sauper, cinéaste, à François Garçon, historien du cinéma
}

\section{Alain Ricard}

\section{(2) OpenEdition}

Journals

Édition électronique

URL : https://journals.openedition.org/eastafrica/603

DOI : $10.4000 /$ eastafrica.603

ISSN : 2790-1076

Éditeur

IFRA - Institut Français de Recherche en Afrique

Édition imprimée

Date de publication : 1 septembre 2008

Pagination : 191-195

ISSN : 2071-7245

\section{Référence électronique}

Alain Ricard, « Pourquoi j'ai accepté de témoigner dans le procès pour diffamation intenté par Hubert Sauper, cinéaste, à François Garçon, historien du cinéma », Les Cahiers d'Afrique de l'Est / The East African Review [En ligne], 40 | 2008, mis en ligne le 07 mai 2019, consulté le 09 décembre 2021. URL : http://journals.openedition.org/eastafrica/603 ; DOI : https://doi.org/10.4000/eastafrica.603

Ce document a été généré automatiquement le 9 décembre 2021

Les Cahiers d'Afrique de l'Est / The East African Review 


\title{
Pourquoi j'ai accepté de témoigner dans le procès pour diffamation intenté par Hubert Sauper, cinéaste, à François Garçon, historien du cinéma
}

\author{
Alain Ricard
}

1 Que vient faire un chercheur dans un procès ? De quelle expertise peut-il se prévaloir? N'est-il que le témoin de moralité, en citoyen ordinaire, ou au contraire essaie-t-il de faire valoir d'autres logiques? J'ai rendu compte dans un texte des réflexions que cette participation m'a suggérées. Je voudrais ici les poursuivre en me posant la question de la place du chercheur.

2 La question de l'interaction entre le filmeur et le filmé, entre le chercheur et le cherché, puisque j'ai utilisé le cinéma comme outil de recherche dans mon travail sur la culture populaire, a été essentielle pour moi. Elle est même devenue une figure de la relation à l'autre dans la forme d'anthropologie culturelle que je me suis mise à pratiquer ; j'ai ainsi rencontré la réflexion de Rouch sur ce travail qu'il a posé dans ses dimensions épistémologiques, et j'ajouterais éthiques puisqu'il s'agit de cela aussi, en termes de déontologie.

3 J'ai abordé ces thèmes dans mon essai sur l'africanisme et j'ai essayé de montrer pourquoi la relation de Rouch à l'Afrique a été pour moi fondatrice, non pas seulement d'un travail cinématographique, mais d'une certaine épistémologie de la recherche, dont on retrouve dans le travail ultérieur d'Edgar Morin les préoccupations et les apports.

4 Ces thèmes sont aujourd'hui développés par quelqu'un comme Jean Louis Comolli dans un enseignement et une réflexion sur le documentaire, qui porte souvent sur l'Afrique et qui demande à dépasser le cadre strict des études cinématographique? 
5 Il inscrit dans une perspective historique sa réflexion sur le documentaire. il note l'importance essentielle de Moi un noir, premier film dans lequel le sujet noir a la parole. Or c'est un sujet africain: la question de l'imposition de pouvoir que constitue l'irruption d'une équipe technique, même réduite ne peut être évacuée. Le journaliste essaie de ramasser ce qu'il peut, il ne fait que passer ; le chercheur essaie de construire, il veut revenir : cela fait une grande différence.

6 La production de documents est au centre de mon travail de chercheur; cette production documentaire est le résultat d'un processus dans lequel les relations de pouvoir (multiples comme Foucault l'a bien montré) se jouent à de multiples niveaux qu'il faut identifier, négocier, et qui font ainsi partie de la construction de la connaissance. J'ai toujours essayé de m'en tenir à ces principes et les règles du cinéma documentaire ont été pour moi des règles de conduite. Certes l'échange est inégal mais nous pouvons quand même parler, écrire voire filmer.

7 Remettre la construction du sens à une phase ultérieure à laquelle le partenaire n'a pas accès, c'est soudain garder le pouvoir essentiel de construction par-devers soi, garder les cartes essentielles dans sa manche. Certes on monte toujours après, et le plan séquence unique ne peut être érigé en principe exclusif; seulement ce montage après peut se faire dans une perspective de respect des interlocuteurs ou au contraire dans une perspective de caricature. Les nuances sont importantes mais on voit bien entre quelles bornes se situe le propos. L'effet Koulechov peut être dévastateur s'il n'obéit pas aux conditions de bonne foi des présupposés implicite du contrat de terrain: vous prenez mon image et ma parole mais vous ne vous moquez pas de moi, vous ne travestissez pas ce que je vous ai dit. Le studio ne peut défaire ce que le terrain a établi.

8 Or Le cauchemar de Darwin m'a frappé dès la première vision. Un phénomène étrange se passait que je ne pouvais décrire mais qui mobilisait mon attention. La présence au générique d'un "field poet » m'a intriguée et j'ai montré ce qu'elle avait induit. Un travail sur les éclairages, des choix de cadrages, construisait toute une dramaturgie spectaculaire et «dramatique » qui ne correspondait pas au propos documentaire : il y avait le filmage, les cadres, les filtres, le travail de l'entretien, une mise en scène dramatique du terrain ; le montage ensuite venait renforcer cette mise en scène par des raccords habiles. Tout cela était très bien fait: trop bien fait? Cette première impression est forte : elle inscrit le propos du film dans une allégorie des rapports nord sud. Aucun jury de festival ne peut être insensible à de bons sentiments aussi dramatiquement exposés. Le chercheur se dit qu'il ne peut en rester là et qu'il faut chercher à comprendre, surtout si son objet de recherche porte sur la mise en textes.

9 Mon malaise personnel face à cette création venait du sentiment confus que les principes sur lesquels mon travail documentaire et anthropologique en Afrique se construisait étaient niés habilement dans ce film.

10 J'en serais resté là si l'occasion ne m'avait pas été donnée de le revoir et si je n'avais pas choisi de m'intéresser au son, qui pour moi est la marque de la durée, matériau propre du cinéma.

11 J'ai ainsi observé, ce qui est devenu la matière de mon témoignage combien les entretiens déformaient les propos des interlocuteurs; j'avais la conviction intime que je pourrais le montrer sur des exemples précis et c'est cela qui s'est produit; au même moment, tout à fait indépendamment une étudiante allemande, Lena Hoernlein, faisait le même constat mû par un même sentiment d'indignation. 
12 En somme le chercheur spécialisé dans la Textwissenschaft, la science des textes, nom que la section 35 du CNRS s'est donné, sous l'influence de nos collègues germanistes, a trouvé dans ce film matière à une critique philologique et dramaturgique.

Il y a une mise en scène de l'Afrique et une manipulation des propos des Africains. La mise en scène n'est pas répréhensible en soi, elle est seulement inadaptée dans le cadre choisi, celui du genre documentaire, et c'est là une question d'histoire de l'art; mais quand la mise en scène se double d'une déformation grave des propos des interlocuteurs, le chercheur en histoire et en science des textes, peut avancer prudemment un témoignage : il peut convoquer l'histoire du genre, et les techniques de « collecte».

14 La question ensuite devient autre : devant le déferlement de ce type de pratique dans les médias télévisuels que faire et pourquoi tant s'émouvoir ? Cette dérive est normale, mais il ne faut pas oublier la question de l'œuvre, de son analyse, de la fabrication du texte du matériau filmé et de sa construction.

15 L'exemple du Cauchemar de Darwin donne à une science toujours en voie de construction, celle de l'analyse du cinéma, quelques éléments empruntés à l'analyse textuelle et à la dramaturgie. Il montre que la conscience historique est une conscience critique, qu'il ne suffit pas de gagner des prix du documentaire pour produire des documents qui permettent à un débat honnête et serein de s'engager. Certes les sujets sont douloureux et considérables, mais doit-on ajouter la tricherie à la violence?

\section{AUTEUR}

\section{ALAIN RICARD}

Directeur de recherche au CNRS, section 35, sciences des textes et des arts. 